\title{
Aptitude à la protéolyse des lactobacilles présents dans les fromages et les lactosérums de fromagerie
}

\author{
par \\ Colette TOURNEUR \\ Laboratoire de Recherche de la Chaire de Technologie (I.N.R.A.) \\ Ecole Nationale Supérieure Agronomique de Grignon (78)
}

\section{Introduction}

Dans les fromages à pâte ferme, pressée ou cuite, les lactobacilles représentent, à une certaine période de la maturation, une fraction importante de la flore microbienne. Les premières semaines d'affinage sont en effet caractérisées par une intense multiplication des ferments lactiques, notamment des lactobacilles. En étudiant la microflore du fromage de Fontine, Bottazzi (1959) met en évidence la disparition des streptocoques après quelques jours d'affinage ; par contre, les lactobacilles se multiplient jusqu'au $50^{\text {me }}$ jour et leur nombre atteint alors $10^{9}$ par gramme. Cette évolution se retrouve dans d'autres variétés de fromages, tels l'Edam (Syrjanen, 1965), le Cheddar (Feagan et Dawson, 1959 ; Johns et Cole, 1959 ; Perry et Shàrpe, 1960), l'Emmental (Sahli et Lehmann, 1959).

A cette période de développement microbien intensif succède généralement une phase pendant laquelle les bactéries lactiques sont lysées et libèrent dans le milieu leurs enzymes intracellulaires ; dans des conditions favorables de $\mathrm{pH}$ et de température, celles-ci sont alors susceptibles d'intervenir dans le processus de dégradation des constituants du caillé, notamment de la caséine. Compte tenu de la durée de l'affinage, du nombre de micro-organismes présents dans la pâte et par suite du potentiel enzymatique qu'ils représentent, les lactobacilles ne seraient pas de simples agents d'acidification intervenant dans le mécanisme de l'égouttage, mais leur rôle dans la formation des constituants de l'arôme et de la saveur pourrait être important.

Les recherches sur l'activité protéolytique des lactobacilles ont été nombreuses au cours de ces dernières années. Les méthodes 
utilisées pour la mettre en évidence sont cependant assez dissemblables et les résultats obtenus sont difficilement comparables. Elles diffèrent notamment par la nature des substrats protéiques et la qualité des extraits enzymatiques. Dolezalek (1966), Tofte Jespersen (1966), Miller et Kandler (1967), Rapp (1969), Lusiani et al. (1971) ne différencient pas phase de production et phase d'activité enzymatiques. L'azote non protéique libéré dans le milieu, constitué principalement d'azote aminé, peut ainsi avoir deux origines : l'excrétion et la protéolyse. Les résultats obtenus par ces différents chercheurs mettent en évidence une activité protéolytique plus marquée chez les espèces thermophiles.

L'importance et la nature de l'activité protéasique endocellulaire de quelques espèces de lactobacilles ont fait l'objet d'un certain nombre de travaux (Amunstad, 1950 ; Baribo et Foster, 1952 ; Brandsaeter et Nelson, 1956 ; Sato et Nakashima, 1965 ; Carini et Ottogalli, 1967 ; Ohmiya et Sato, 1968 ; Ducastelle et Lenoir, 1969). Les conditions optimales d'activité ont été recherchées : Baribo et Foster (1952), Brandsaeter et Nelson (1956) notent, chez Lactobacillus casei, un maximum d'activité dans une zone se situant vers $\mathrm{pH} 6,0$, la température la plus favorable étant voisine de $40-45^{\circ} \mathrm{C}$. Ces observations sont en accord avec celles de Christophersen et Thiele (1952) sur Lactobacillus helveticus et Lactobacillus lactis. Plus récemment Ducastelle et Lenoir (1969) font état de résultats comparables chez trois souches de Lactobacillus plantarum isolées de Saint-Paulin, lesquelles présentent une activité optimale à un $\mathrm{pH}$ voisin de 5,5 . Les différentes souches du genre Lactobacillus possèdent donc un système protéasique endocellulaire, caractérisé par une activité maximale à des valeurs de $\mathrm{pH}$ très proches de celles des fromages en cours de maturation. En outre, elles semblent dégrader préférentiellement la caséine $\alpha$ (Annibaldi, 1969 ; Ohmiya et Sato, 1969), et plus particulièrement la caséine $\alpha_{\text {s. }}$.

Ces recherches portaient toutefois sur un petit nombre de souches et d'espèces. Elles ne reflètent probablement pas l'aptitude à la protéolyse de toutes les espèces du genre Lactobacillus susceptibles d'être rencontrées dans les produits laitiers.

Notre étude répond donc à trois objectifs :

- Réunir une collection de souches de lactobacilles isolées de produits de fromagerie : caillés, lactosérums et fromages à maturation lente.

- Tester l'aptitude à la protéolyse de cette population dans des conditions bien définies de production, d'extraction et de digestion enzymatiques et déterminer si cette aptitude est un caractère d'espèce ou de souche.

- Préciser la nature de l'activité protéolytique par détermination de la qualité des produits libérés. 


\section{Constitution d'une collection de souches}

\section{1) ORIGINE DES ÉCHANTILLONS}

Trois types de produits différents ont été analysés :

- Des fromages affinés, prêts à la commercialisation et prélevés sur les lieux mêmes de la fabrication. Parmi eux :

- sept pâtes fermes : Emmental, Fribourg, Fontine, Parmesan, Provolone, Cantal (trois échantillons) et Laguiolle ;

- cinq pâtes pressées : Chevrotin, Reblochon, fromage de brebis des Pyrénées, Saint-Nectaire et Mimolette ;

- Quatre caillés de pâtes pressées, choisis à divers stades de fabrication :

- Reblochon : un échantillon sortie saloir, une échantillon ayant 10 jours de cave ;

- Tome de Savoie : un échantillon sortie saloir ;

- Chevrotin : un échantillon sortie saloir.

- Dix-sept lactosérums de fromagerie de Gruyère, provenant de 13 fruitières de la région de Poligny (Jura), dont 12 n'utilisent jamais de ferments sélectionnés. Cinq fabrications avaient été réalisées à l'aide d'un levain sur lactosérum cru et les autres avec une présure naturelle sur recuite ou sur lactosérum.

\section{2) Protocole expérimental} caillés

2.1. Dénombrement et isolement à partir des fromages et des

La partie externe du fromage est éliminée sur $1 \mathrm{~cm}$ d'épaisseur. Une partie aliquote de l'intérieur est alors broyée dans un mortier et $10 \mathrm{~g}$ de pâte homogénéisée sont mis en suspension (AtoMix MSE) dans $90 \mathrm{ml}$ d'une solution stérile de citrate de sodium à $2 \mathrm{p} .100$. Des dilutions décimales de cette suspension sont réalisées à l'aide de solution de Ringer au $1 / 4$ et $1 \mathrm{ml}$ de chaque dilution est ensemencée dans la masse d'un milieu gélosé coulé en boîte de Pétri. Les germes totaux sont dénombrés sur Tryptone-agar (1), les lactobacilles sur gélose MRS (de Man et al., 1960) et sur gélose Rogosa modifiée (Mabbitt et Zielinska, 1956). L'incubation a lieu à $30^{\circ} \mathrm{C}$ pendant 2 et 3 jours pour les germes totaux et le milieu MRS, 4 et $8 \mathrm{j}$ pour le milieu Rogosa modifié.

Pour chaque échantillon, 5 à 10 colonies sont prélevées sur les milieux MRS ou Rogosa modifié et repiquées sur milieu MRS. Après purification, les souches sont conservées, d'une part sur lait tournesolé à $-30^{\circ} \mathrm{C}(1 \mathrm{ml}$ de culture de $16 \mathrm{~h}$ pour $9 \mathrm{ml}$ de lait), d'autre part sur gélose MRS-caséine (2) à $+4^{\circ} \mathrm{C}$.

(1) Tryptone-agar : tryptone : $5 \mathrm{~g}$; extrait de levure : $3 \mathrm{~g}$; glucose : $1 \mathrm{~g}$; eau q.s.p. $1000 \mathrm{ml}$; gélose : $20 \mathrm{~g}$; pH 7 ; stériliser $120^{\circ} \mathrm{C}$ pendant $20 \mathrm{mn}$.

(2) Gélose MRS-caséine : gélose MRS additionnée de caséinate à 2 p. 100. 


\subsection{Isolement à partir des lactosérums et des yaourts}

Avant isolement, on procède à un enrichissement des échantillons par culture sur lait stérilisé, sur bouillon APT (Evans et Niven, 1951 ) et sur bouillon MRS, incubés à $40^{\circ} \mathrm{C}$ pendant $16 \mathrm{~h}$. A partir de chacun de ces milieux, des isolements sont effectués à la surface des milieux gélosés MRS et Rogosa modifié ; chaque isolement est réalisé en double exemplaire : une boîte est placée en aérobiose à $40^{\circ} \mathrm{C}$, l'autre est incubée en atmosphère d'azote à $42^{\circ} \mathrm{C}$.

Après 3,4 et $8 \mathrm{j}$ d'incubation suivant les milieux, quelques colonies sont prélevées, purifiées et conservées par des procédés identiques à ceux utilisés pour les souches isolées des fromages.

\subsection{Identification}

L'identification des souches est faite selon les critères préconisés par Sharpe (1962). Les températures d'incubation retenues ont été :

- $30^{\circ} \mathrm{C}$ pour les souches isolées des fromages et caillés.

- $40^{\circ} \mathrm{C}$ pour celles provenant des lactosérums (recherche spécifique des bactéries thermophiles).

Les caractères d'identification suivants ont été étudiés :

- Morphologie : Un examen morphologique est effectué par coloration de Gram sur une culture de $18 \mathrm{~h}$ en bouillon MRS.

- Production de catalase : Une colonie est prélevée sur gélose MRS et dissociée dans une goutte d'eau oxygénée à 10 volumes; le dégagement d'oxygène qui peut se produire est l'indice d'une souche possédant une catalase.

- Croissance à différentes températures : Ce test est réalisé sur bouillon MRS, porté préalablement à la température désirée : $15^{\circ} \mathrm{C}\left( \pm 0,5^{\circ} \mathrm{C}\right)$ et $45^{\circ} \mathrm{C}\left( \pm 0,5^{\circ} \mathrm{C}\right)$. La croissance est appréciée par turbidimétrie après $2 \mathrm{j}$ à $45^{\circ} \mathrm{C}$ et $8 \mathrm{j}$ à $15^{\circ} \mathrm{C}$.

- Acidité titrable : Après $8 \mathrm{j}$ de culture sur lait écrémé stérilisé par autoclavage $15 \mathrm{mn}$ à $115^{\circ} \mathrm{C}$, l'acidité est titrée sur $10 \mathrm{~g}$ de lait, à l'aide de soude $\mathrm{N} / 9$, en présence de phénolphtaléine. Elle est exprimée en grammes d'acide lactique pour $100 \mathrm{~g}$ de lait.

- Désamination de l'arginine : Cette activité enzymatique est mise en évidence sur bouillon MRS à 2 p. 100 de glucose (bouillon MRS classique) et sur bouillon MRS à 1 p. 100 de glucose, additionnés de 0,3 p. 100 de L-arginine monochlorhydrate. Après incubation pendant une semaine, l'ammoniaque libéré est recherché à l'aide du réactif de Nessler.

- Croissance en présence de concentrations croissantes de teepol : Le bouillon MRS est additionné de différentes quantités de teepol $(0,2-0,4-0,7$ p. 100). La croissance est appréciée par mesure de la turbidité du milieu après 2,4 et $6 \mathrm{j}$ de culture.

- Fermentation des sucres : Le milieu de base est le milieu MRS, exempt de glucose et d'extrait de viande, additionné de rouge 
de chlorophénol à 0,004 p. 100. Des solutions stériles des sucres suivants sont ajoutées au milieu après stérilisation : arabinose, cellobiose, galactose, lactose, raffinose, rhamnose, saccharose, salicine, tréhalose et xylose, la concentration finale étant 0,5 p. 100 (de Man et al., 1960). L'acidification est appréciée après 2, 4 et $6 \mathrm{j}$ d'incubation.

- Hydrolyse de l'esculine : L'aptitude à hydrolyser l'esculine est décelée par formation d'un précipité noir dans un bouillon MRS modifié contenant 0,5 p. 100 d'esculine et 0,05 p. 100 de citrate ferrique ammoniacal. La lecture est faite après 3 et $6 \mathrm{j}$ de croissance.

- Production de gaz à partir du glucose : Le milieu de base est le milieu MRS, enrichi en glucose ( 5 p. 100) et additionné de gélatine à $20 \mathrm{p} .100$. Le milieu, réparti dans des tubes à essais à raison de $10 \mathrm{ml}$ par tube, est porté à $45^{\circ} \mathrm{C}$ et ensemencé à l'aide d'un inoculum important (culot de centrifugation d'une culture de $16 \mathrm{~h}$ sur $10 \mathrm{ml}$ de bouillon MRS). Après répartition de l'inoculum au sein du milieu et solidification de celui-ci, de la paraffine stérile est coulée à la surface sur une épaisseur de $1 \mathrm{~cm}$. La fermentation avec formation de gaz se manifeste par le soulèvement de la plaque de paraffine. La lecture est faite quotidiennement pendant $12 \mathrm{j}$.

- Configuration stéréochimique de l'acide lactique : Elle est déterminée par appréciation du pourcentage d'acide $L(+)$ lactique formé par rapport à l'acide lactique total, dans une culture de $8 \mathrm{j}$ sur lait écrémé. L'acide lactique total est dosé colorimétriquement par la méthode de Steinsholt et Calbert (1960) et l'acide L (+) lactique par dosage enzymatique selon Mattson (1965).

\section{3) Résultats}

\section{culture \\ 3.1. Comparaison des croissances sur les différents milieux de}

3.1.1. Dénombrement sur les différents milieux : Les examens ont été réalisés sur tous les fromages étudiés, à l'exception du Parmesan du Saint-Nectaire et du Laguiolle (tab. 1).

Avec 50 p. 100 des échantillons analysés, la population sur milieu MRS est aussi importante que celle dénombrée sur Tryptone-agar, milieu utilisé généralement pour l'évaluation de la flore totale et considéré comme peu favorable à la croissance des germes exigeants sur le plan nutritionnel tels les lactobacilles.

Par contre la flore capable de se multiplier sur le milieu Rogosa modifié est nettement plus réduite, et l'importance de cette microflore varie avec les divers types de fromages affinés.

\subsubsection{Influence des conditions expérimentales.}

- Influence du milieu : Afin de vérifier l'aptitude des lactobacilles à croître sur ces différents milieux, des dénombrements ont 
Dénombrement de la microflore des fromages sur différents milieux de culture*

(Les résultats sont exprimés en millions de micro-organismes par gramme de fromage)

\begin{tabular}{|c|c|c|c|}
\hline & \multicolumn{3}{|c|}{ Milieux de culture } \\
\hline & Tryptone-agar (TA) & MRS & Rogosa modifié ( $\mathrm{Rog}$ ) \\
\hline Emmental & 30 & 30 & 10 \\
\hline Fribourg & 13 & 13 & 10 \\
\hline Fontine & 2500 & 2000 & 20 \\
\hline Provolone & 100 & 50 & 30 \\
\hline Cantal & 2100 & 1250 & 4000 \\
\hline Chevrotin (affiné) & 1600 & 1600 & 7 \\
\hline Chevrotin (saloir) & 2200 & 175 & 1 \\
\hline Reblochon (affiné) & 4000 & 4000 & 100 \\
\hline Reblochon (cave) & 4000 & 4000 & 50 \\
\hline Reblochon (saloir) & 3000 & 3000 & 25 \\
\hline Fromage des Pyrénées & 90 & 30 & 8 \\
\hline Mimolette & 1,5 & 0,5 & 0,15 \\
\hline Tome (saloir) & 2000 & 300 & 15 \\
\hline
\end{tabular}

* Conditions des essais : - température de croissance $=30^{\circ} \mathrm{C}$

- durée d'incubation $=$ TA $: 2 \mathrm{j} ;$ MRS $: 3 \mathrm{j}$;

Rog: 4 et $8 \mathrm{j}$. 
été réalisés, dans des conditions identiques à celles utilisées pour les fromages, sur des cultures pures de lactobacilles types, développées sur lait écrémé pendant $18 \mathrm{~h}$ (tab. 2).

L'examen des résultats met en évidence les faits suivants :

- la plupart des espèces se multiplient activement sur Tryptoneagar, mais le milieu MRS se révèle cependant plus favorable, notamment pour la croissance des lactobacilles thermophiles ;

- le milieu de Rogosa modifié est inhibiteur pour la plupart des micro-organismes testés. Cette constatation est particulièrement nette pour les lactobacilles thermophiles et l'espèce $L$. plantarum.

- Influence de la température : Les dénombrements sur fromages ayant été réalisés après incubation à $30^{\circ} \mathrm{C}$, l'aptitude des lactobacilles thermophiles à se multiplier à cette température sur les milieux choisis devait être vérifiée. Dans l'expérience précédente, les dénombrements ont donc été effectués à trois températures : $30^{\circ} \mathrm{C}$, $37^{\circ} \mathrm{C}$ et $45^{\circ} \mathrm{C}$ (tab. 2). Les résultats obtenus montrent que la température de $30^{\circ} \mathrm{C}$, moins favorable à la croissance des lactobacilles thermophiles que la température de $37^{\circ} \mathrm{C}$, peut être un facteur limitant pour le dénombrement et la recherche de ces micro-organismes.

\subsection{Isolement et identification des lactobacilles}

3.2.1. Caractères des souches isolées à partir des fromages : 171 souches ont été isolées à partir des milieux MRS et Rogosa modifié. Une première sélection a été effectuée par examen morphologique, coloration de Gram et recherche de la catalase, à la suite de laquelle seules ont été retenues les bactéries catalase négative, gram positif, en forme de bâtonnet. Ont été rejetées 40 souches, de morphologie atypique, mais dont aucune n'avait cependant l'aspect caractéristique des coques ; 70 p. 100 des micro-organismes ainsi éliminés avaient été prélevés sur le milieu Rogosa modifié.

Les 131 souches sélectionnées ont été identifiées. Toutes appartiennent aux groupes Streptobacterium et Betabacterium selon OrlaJensen ; aucun lactobacille thermophile n'a été trouvé. Deux espèces prédominent : L. casei et L. plantarum. Parmi les lactobacilles hétérofermentaires, certaines souches n'ont pu être identifiées selon les critères de Rogosa et Sharpe (1959).

La répartition des espèces isolées est la suivante :

- 57 L. casei.

- 50 L. plantarum.

- 24 lactobacilles hétéro-fermentaires dont 2 L. buchneri,

13 L. brevis et 9 souches indéterminées.

3.2.2. Caractères des souches isolées à partir des lactosérums et des yaourts : 169 colonies ont été prélevées sur les milieux MRS placés en aérobiose ou en anaérobiose et sur le milieu Rogosa 
Influence des milieux de culture et de la température d'incubation sur la croissance de diverses espèces de lactobacilles* (les résultats sont exprimés en millions de micro-organismes par $\mathrm{ml}$ de culture sur lait stérilisé)

\begin{tabular}{|c|c|c|c|c|c|}
\hline \multirow{2}{*}{$\begin{array}{l}\text { Température } \\
\text { d'incubation }\end{array}$} & \multirow{2}{*}{ Souches } & \multicolumn{4}{|c|}{ Milieux de culture } \\
\hline & & Tryptone-agar (TA) & MRS & Rogosa & modifié (Rog) \\
\hline $\begin{array}{ll}30^{\circ} & \mathrm{C} \\
30^{\circ} & \mathrm{C} \\
30^{\circ} & \mathrm{C} \\
30^{\circ} & \mathrm{C} \\
30^{\circ} & \mathrm{C} \\
30^{\circ} & \mathrm{C} \\
30^{\circ} & \mathrm{C} \\
37^{\circ} & \mathrm{C} \\
45^{\circ} & \mathrm{C} \\
30^{\circ} & \mathrm{C} \\
37^{\circ} & \mathrm{C} \\
45^{\circ} & \mathrm{C}\end{array}$ & $\begin{array}{l}\text { L. casei } \mathrm{C}_{7} \\
\text { L. casei } 2 \\
\text { L. casei } \mathrm{L}_{2-4} \\
\text { L. buchneri } \mathrm{E}_{7} \\
\text { L. brevis } \mathrm{C}_{5} \\
\text { L. plantarum } \mathrm{N}_{5} \\
\text { L. helveticus CNRZ } 240 \\
\quad " \\
\quad " \\
\text { L. bulgaricus CNRZ } 208 \\
\quad "\end{array}$ & $\begin{array}{r}1900 \\
1000 \\
2600 \\
2400 \\
5400 \\
5000 \\
2 \\
100 \\
150 \\
30 \\
150 \\
100\end{array}$ & $\begin{array}{r}2400 \\
1300 \\
1900 \\
2200 \\
4500 \\
8000 \\
3 \\
250 \\
100 \\
600 \\
500 \\
200\end{array}$ & & $\begin{aligned} 1000 \\
100 \\
300 \\
1000 \\
1500 \\
100 \\
<0,1 \\
<0,1 \\
<0,1 \\
<0,1 \\
<0,1 \\
<0,1\end{aligned}$ \\
\hline
\end{tabular}

* Conditions des essais : - dénombrement effectué sur des cultures de $18 \mathrm{~h}$ sur lait écrémé stérilisé.

- durée d'incubation $=$ TA $: 2 \mathrm{j} ;$ MRS $: 3 \mathrm{j}$;

Rog : 4 et $8 \mathbf{j}$. 
modifié incubé en anaérobiose ; aucune croissance n'a pu être obtenue sur le même milieu placé en aérobiose.

Après examen morphologique et recherche de la catalase, 63 souches isolées de lactosérums et 3 souches isolées de yaourts ont été retenues pour identification.

Toutes sont des lactobacilles thermophiles appartenant aux groupes Thermobacterium et Betabacterium selon Orla-Jensen ; ce résultat s'explique en partie par la température d'incubation choisie. Quelques souches ne s'apparentent à aucune des espèces définies par Rogosa et Sharpe (1959) ; elles en diffèrent notamment par leur aptitude à fermenter un ou deux sucres.

Ont été ainsi isolées :

- parmi les lactobacilles hétéro-fermentaires : $14 \mathrm{~L}$. fermenti,

- parmi les lactobacilles homo-fermentaires : 8 L. acidophilus, 1 L. delbruecki, 2 L. salivarius, 16 L. helveticus, 13 L. jugurti (ou L. bulgaricus) et 12 lactobacilles indéterminés.

\section{4) Discussion}

Le nombre réduit des échantillons analysés ne permet pas de faire une étude écologique de la flore lactobacilles des fromages. L'objectif visé était uniquement la mise en place d'une collection de ces micro-organismes à partir de produits fromagers dans lesquels ils sont susceptibles d'intervenir.

L'examen des résultats obtenus sur les fromages (dénombrement et identification) confirme les propriétés sélectives différentes des milieux de culture utilisés et permet de constater l'absence de lactobacilles thermophiles dans la microflore isolée.

Deux questions peuvent alors se poser :

- Quelle est l'importance réelle des lactobacilles dans la population dénombrée sur ces trois milieux ?

- L'absence de lactobacilles thermophiles est-elle la conséquence des conditions expérimentales choisies ou l'indice d'une réelle disparition de ces espèces dans les fromages étudiés ?

L'analyse des résultats figurant dans le tableau 1 et la connaissance des aptitudes nutritionnelles des milieux de culture (Mabbitt et Zielinska, 1956 ; de Man et al., 1960) permettaient de formuler, à priori, 1'hypothèse suivante : le milieu MRS, non sélectif, permet de dénombrer, outre les lactobacilles, une partie importante de la population des fromages; le milieu sélectif de Rogosa modifié donnerait seul une image exacte de l'importance des lactobacilles dans la microflore totale. Ces micro-organismes représenteraient alors une fraction très faible de la population.

Deux faits infirment cette hypothèse : l'analyse des dénombrements réalisés sur des cultures pures et l'identification des microorganismes isolés. 
- Le milieu de Rogosa modifié est inhibiteur, à des degrés variables, pour tous les lactobacilles, en particulier pour les espèces thermophiles (tab. 2).

- Environ 80 p. 100 des micro-organismes prélevés au hasard sur le milieu MRS ont été identifiés au genre Lactobacillus. Les souches éliminées se caractérisaient par une morphologie atypique ; elles pouvaient cependant appartenir à ce genre car des lactobacilles atypiques ont été isolés des viandes par Reuter (1970) et Sharpe (1962) a constaté un hétéromorphisme accentué fréquent chez les lactobacilles, en fonction des conditions de culture.

La microflore se multipliant sur gélose MRS étant essentiellement constituée de lactobacilles, ceux-ci représentent donc la population dominante des fromages affinés analysés. Comme il a été observé sur quelques types de fromages, le Cheddar (Naylor et Sharpe, 1958) et l'Emmental (Sahli et Lehmann, 1959), il se produit sans doute, au cours de la maturation, une évolution de la flore qui se traduit par une augmentation du nombre de lactobacilles et une disparition des streptocoques.

Les expériences réalisées avec $L$. helveticus et $L$. bulgaricus (tab. 2) nous permettent de répondre au problème posé par l'absence de germes thermophiles dans les isolements effectués à partir des fromages. Nous constatons que certains de ces micro-organismes peuvent se multiplier à $30^{\circ} \mathrm{C}$ sur les milieux utilisés. Leur croissance est sans doute plus lente mais il est peu probable que le facteur température d'incubation soit à l'origine d'une inhibition telle que, sur les 131 souches isolées, aucune n'appartienne au groupe des lactobacilles thermophiles. Au cours de la maturation des fromages à pâte cuite, il est plus vraisemblable que la microflore thermophile des levains disparaît progressivement alors que les lactobacilles mésophiles se multiplient activement et prédominent en fin d'affinage.

La recherche des lactobacilles thermophiles devait donc se faire à partir de produits provenant d'une fabrication fromagère à ses débuts, caillés ou lactosérums. C'est ainsi qu'à partir des lactosérums de fromageries de gruyère et en mettant en œuvre une température d'incubation plus élevée, il a été possible de sélectionner des lactobacilles thermophiles, homo et hétéro-fermentaires, appartenant à des espèces très variées.

\section{Détermination de l'aptitude des lactobacilles à la production d'enzymes protéolytiques}

\section{1) ORIGINE ET CHOIX DES SOUCHES}

L'aptitude à la protéolyse a été étudiée sur 103 souches choisies parmi les 197 qui composent la collection. Elles ont été sélectionnées selon trois critères : 
- Leur identité. fait).

- Leur origine (échantillon à partir duquel l'isolement a été

- Leur pouvoir acidifiant différent dans le cas de deux souches de même espèce provenant du même échantillon.

La répartition, la nature et l'origine des souches testées figurent dans le tableau 3.

TABLEAU 3. - Identité et origine des souches testées

\begin{tabular}{|c|c|c|}
\hline Espèces & $\begin{array}{l}\text { Nombre de } \\
\text { souches testées }\end{array}$ & Provenant de \\
\hline $\begin{array}{l}\text { Streptobacterium : } \\
\text { L. casei } \\
\text { L. plantarum }\end{array}$ & $\begin{array}{l}26 \\
23\end{array}$ & $\begin{array}{l}15 \text { fromages } \\
11 \text { fromages }\end{array}$ \\
\hline $\begin{array}{l}\text { Thermobacterium : } \\
\text { L. acidophilus } \\
\text { L. jugurti } \\
\text { L. helveticus } \\
\text { L. salivarius } \\
\text { L. delbrueki } \\
\text { Lactobacilles indéterminés }\end{array}$ & $\begin{array}{l}7 \\
9 \\
8 \\
2 \\
1 \\
6\end{array}$ & $\begin{array}{l}6 \text { lactosérums } \\
9 \text { lactosérums } \\
5 \text { lactosérums } \\
1 \text { lactosérum } \\
1 \text { lactosérum } \\
3 \text { lactosérums et } \\
3 \text { yaourts }\end{array}$ \\
\hline $\begin{array}{l}\text { Betabacterium : } \\
\text { L. fermenti } \\
\text { L. brevis } \\
\text { L. buchneri } \\
\text { Lactobacilles indéterminés }\end{array}$ & $\begin{array}{r}11 \\
4 \\
2 \\
4\end{array}$ & $\begin{array}{l}10 \text { lactosérums } \\
2 \text { fromages } \\
2 \text { fromages } \\
4 \text { fromages }\end{array}$ \\
\hline Total & 103 & \\
\hline
\end{tabular}

\section{2) Protocole expérimental}

\subsection{Conditions de production}

Chaque souche est ensemencée dans un bouillon MRS, réparti en ballon de 21 à raison de 11 de milieu par ballon, l'inoculum étant constitué par $10 \mathrm{ml}$ d'une culture de $16 \mathrm{~h}$ sur bouillon MRS.

Les milieux sont placés à l'étuve pendant 3 jours, à $30^{\circ} \mathrm{C}$ pour les lactobacilles mésophiles, à $40^{\circ} \mathrm{C}$ pour les souches thermophiles. Pendant cette période, le $\mathrm{pH}$ du milieu est ajusté, toutes les 12 heures, à une valeur voisine de 6,2 , à l'aide d'une solution de soude $4 \mathrm{~N}$. L'évolution du $\mathrm{pH}$ est notée avant et après chaque ajustement. 
fig. 1

Schéma de préparation des extraits enzymatiques

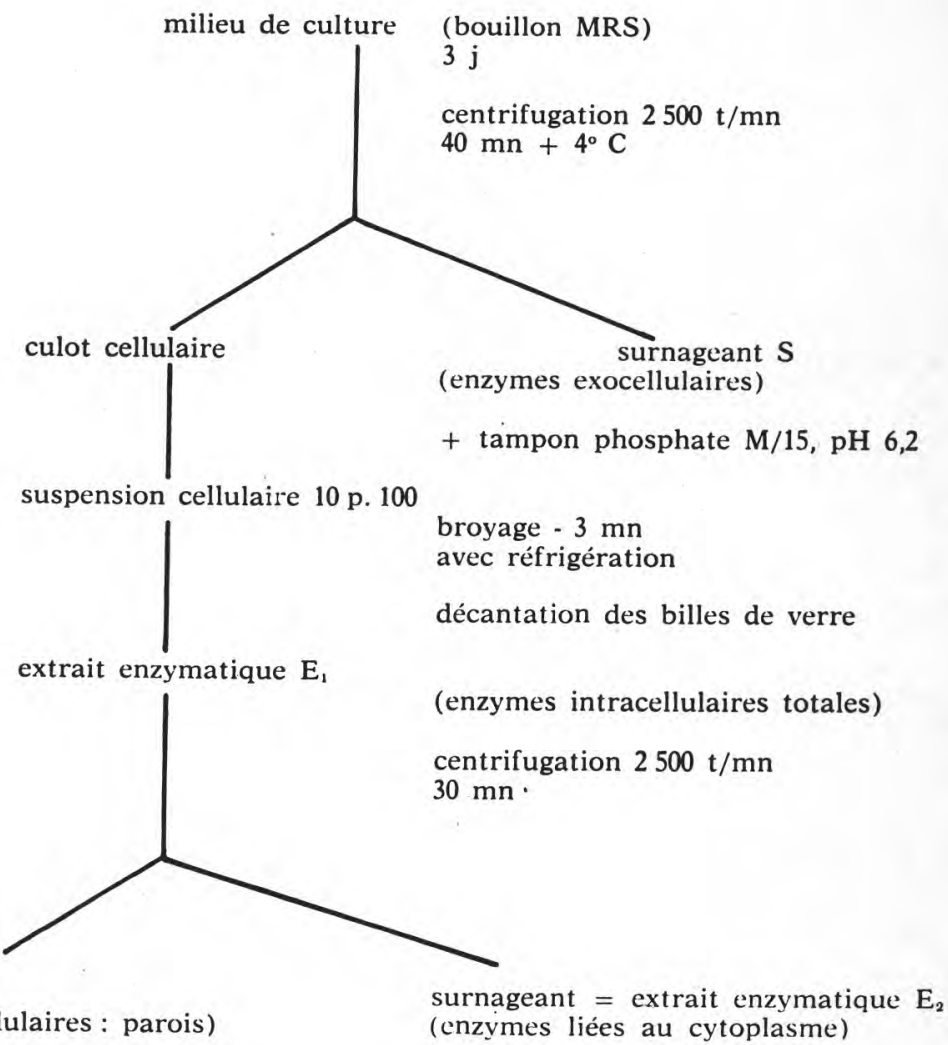

culot

(débris cellulaires : parois) (enzymes liées au cytoplasme)

\subsection{Détermination de l'activité enzymatique}

2.2.1. Préparation des extraits enzymatiques (fig. 1) : En fin d'incubation, les cellules sont séparées du milieu de culture par centrifugation à $2500 \mathrm{t} / \mathrm{mn}$ pendant $40 \mathrm{mn}$ à $+4^{\circ} \mathrm{C}$.

Le surnageant de centrifugation $\mathrm{S}$ représente l'extrait enzymatique exocellulaire.

Le culot est lavé deux fois de suite par dispersion dans une solution de tampon phosphate $\mathrm{M} / 15$ ajustée à $\mathrm{pH}$ 6,2, puis centrifugé. Il est ensuite pesé et une quantité suffisante de tampon est ajoutée de façon à obtenir une suspension cellulaire à $10 \mathrm{p} .100$ (poids/poids), dont l'homogénéisation est réalisée à l'aide de l'appareil Ultra-Turrax. Le broyage des cellules est effectué avec l'appareil Braun (type 2876 ), en flacon de $60 \mathrm{ml}$ contenant $50 \mathrm{~g}$ de billes de 
verre de 0,10-0,11 $\mathrm{mm}$ de diamètre. La durée du broyage est de $3 \mathrm{mn}$ et le refroidissement. est assuré par détente d'anhydride carbonique liquide.

Par simple décantation des billes de verre, on obtient l'extrait enzymatique endocellulaire $E_{1}$, liquide opalescent contenant les débris des parois cellulaires.

Cet extrait est ensuite centrifugé à $2500 \mathrm{t} / \mathrm{mn}$ pendant $30 \mathrm{mn}$. Le surnageant de centrifugation constitue l'extrait enzymatique $\mathrm{E}_{2}$, exempt de particules cellulaires.

2.2.2. Conditions de digestion : L'activité protéolytique des extraits est déterminée sur un substrat à base de caséine : caséine Hammarsten (NBC) : $25 \mathrm{~g}$; citrate de sodium 0,02 M q.s.p. $1000 \mathrm{ml}$, le mélange étant ajusté à $\mathrm{pH}$ 7,0 par addition de soude environ $\mathrm{N}$. Ce substrat est stérilisé à $120^{\circ} \mathrm{C}$ pendant $20 \mathrm{mn}$.

Le mélange de digestion, placé en tubes à essais, a la composition suivante :

$\begin{array}{ll}\text { substrat caséine } & 2,5 \mathrm{ml} \\ \text { tampon mixte pH 5,5 (3) } & 0,5 \mathrm{ml} \\ \text { solution de merthiolate } 0,25 \mathrm{p} \cdot 100 & 2 \text { gouttes } \\ \left.\text { extrait enzymatique ( } \mathrm{E}_{1}-\mathrm{E}_{2}-\mathrm{S}\right) & 2 \mathrm{ml}\end{array}$

Dans ces conditions, le $\mathrm{pH}$ du milieu de digestion est 6,3.

Les mélanges réactionnels sont maintenus dans un bain-marie à $40^{\circ} \mathrm{C}$ pendant $16 \mathrm{~h}$.

2.2.3. Mesure de l'activité enzymatique : Le mélange de digestion est déprotéiné par addition d'un volume égal d'acide trichloracétique à 4 p. 100. Sur le filtrat, les composés azotés non protéiques solubles sont dosés selon deux méthodes :

- Les acides aminés à groupement phénolique, libres ou engagés dans des peptides, sont estimés avec le réactif de Folin et Ciocalteu (1927), selon la méthode de Anson (1938). A $1 \mathrm{ml}$ de filtrat sont ajoutés $6,5 \mathrm{ml}$ d'eau, $2 \mathrm{ml}$ d'une solution de carbonate de sodium à 15 p. $100,0,5 \mathrm{ml}$ de réactif de Folin. La coloration est développée pendant $5 \mathrm{~h}$ à température ambiante et son intensité est mesurée à la longueur d'onde de $650 \mathrm{~m} \mu$, avec un colorimètre Lumétron.

L'azote non protéique est exprimé en $\mu \mathrm{g}$ de tyrosine par $\mathrm{ml}$ d'extrait enzymatique et par heure de digestion, en se référant à une courbe étalon de tyrosine.

- Les groupements $-\mathrm{NH}_{2}$ libres sont dosés par coloration à la ninhydrine selon la méthode de Moore et Stein (1948) modifiée par la Cie Technicon (1968). La coloration est développée et mesurée

(3) Tampon mixte : acétate-borate-phosphate 0,25 $\mathrm{M}$, ajusté au pH désiré par addition de soude ou d'acide sulfurique $5 \mathrm{~N}$. 
automatiquement avec l'appareil Auto-Analyseur Technicon, à la longueur d'onde de $570 \mathrm{~m} \mu$, la vitesse de prélèvement étant de 20 échantillons à l'heure.

Les résultats sont exprimés en $\mu \mathrm{g}$ de leucine par $\mathrm{ml}$ d'extrait enzymatique et par heure de digestion, par comparaison avec une courbe d'étalonnage de leucine.

Des mélanges de digestion non incubés, traités dans des conditions identiques, constituent les blancs de coloration.

\section{3) Résultats}

\subsection{Mise au point des conditions expérimentales}

Au cours d'essais préliminaires portant sur l'étude de trois à sept souches appartenant à des espèces différentes, quelques facteurs pouvant avoir une incidence au niveau de la production enzymatique, de la préparation des extraits, de la digestion enzymatique et du dosage des produits libérés ont été étudiés.

- Au niveau de la production de cellules et d'enzymes :

L'addition de caséinate au milieu de culture, à une concentration de 2 p. 100 , ne modifie pas de façon significative la production d'enzymes.

Le réajustement périodique du $\mathrm{pH}$ pendant la phase de production à une valeur voisine de 6,0, a une influence variable suivant les souches ; sur les cinq souches testées, deux ( $L$. casei et $L$. brevis) sont sensibles à l'acidification du milieu ; leur activité enzymatique est diminuée de moitié si la croissance s'effectue dans un milieu à pH non contrôlé. Les trois autres ( $L$. plantarum, L. casei et $L$. buchneri) montrent une activité identique quel que soit le $\mathrm{pH}$ final du milieu.

L'influence du temps d'incubation a été déterminée après 2 - 3 5 - 7 et $9 \mathrm{j}$ de culture à $\mathrm{pH}$ contrôlé. L'activité protéolytique est maximale après 2 à $3 \mathrm{j}$ d'incubation; elle régresse ensuite pour devenir pratiquement nulle au bout de $9 \mathrm{j}$, sans qu'apparaisse une modification du poids de cellules récoltées.

- Au niveau de la préparation de l'extrait endocellulaire :

L'efficacité du broyage a été testée par dénombrement, sur gélose MRS, des cellules de la suspension cellulaire, avant et après broyage. 99,9 p. 100 des cellules sont détruites dans les conditions expérimentales utilisées.

La reproductibilité du broyage a été estimée en comparant l'activité protéasique de six broyats (extraits $\mathrm{E}_{1}$ et $\mathrm{E}_{2}$ ) provenant de la même suspension cellulaire. Les résultats obtenus sont reproductibles ; les activités protéolytiques des six extraits $E_{1}$ sont situés dans l'intervalle $\bar{x} \pm \sigma \mathrm{t}=15,3 \pm 0,75 \mu \mathrm{g}$ de tyrosine par ml d'extrait 
et par heure de digestion, celles des extraits $E_{2}$, dans l'intervalle $\bar{x} \pm \sigma \mathrm{t}=10 \pm 0,7 \mu \mathrm{g}$ de tyrosine par ml d'extrait et par heure de digestion.

- Au niveau des conditions de digestion :

L'influence du $\mathrm{pH}$ du mélange de digestion a été déterminée à deux valeurs : $\mathrm{pH}$ 6,3 et $\mathrm{pH}$ 5,8. Les différences d'activité observées sont très légères et toujours inférieures à 5 p. 100 .

L'influence du temps de digestion a été étudiée dans l'intervalle 0 - $16 \mathrm{~h}$, à la température de $40^{\circ} \mathrm{C}$, au $\mathrm{pH}$ de 6,3 et en présence de $2 \mathrm{ml}$ d'extrait enzymatique. Les résultats montrent que la quantité de produits libérés est, dans la plupart des cas, proportionnelle au temps de digestion.

L'influence de la quantité d'enzymes a été estimée en faisant agir des volumes variables d'extrait, entre 0 et $2 \mathrm{ml}$, à $\mathrm{pH} 6,3$, la température de digestion étant $40^{\circ} \mathrm{C}$ et le temps de $16 \mathrm{~h}$. Il y a un rapport direct entre la quantité de tyrosine libérée et la quantité d'enzymes.

- Au niveau de la détermination des produits libérés :

La nature de l'activité des lactobacilles a été étudiée, sur vingt souches, en dosant, par la méthode de Anson, la tyrosine libérée dans les filtrats trichloracétiques à 12 p. 100 et à 2 p. 100 . Les résultats obtenus sont identiques dans les deux filtrats, ce qui permet de penser que les lactobacilles libèrent des composés azotés de faible poids moléculaire et possèdent une activité protéolytique de type exopeptidasique.

\subsection{Aptitude à la protéolyse des souches de collection}

3.2.1. Activité exocellulaire : L'activité enzymatique du surnageant de centrifugation $\mathrm{S}$ a été recherchée sur quatre souches mésophiles d'espèces différentes, selon la méthode de Anson.

Aucune activité exocellulaire n'a pu être mise en évidence. Notons toutefois que le milieu de culture non ensemencé étant luimême riche en acides aminés aromatiques, la valeur du témoin est très élevée et une faible activité ne peut être mise en évidence.

3.2.2. Activité endocellulaire cytoplasmique : Dans les conditions de préparation utilisées, l'extrait enzymatique $\mathrm{E}_{2}$ est libre de débris cellulaires. Il ne contient donc que les enzymes d'origine cytoplasmique (Pollock, 1962).

L'aptitude à la protéolyse des 103 souches sélectionnées a été déterminée par la mesure de la quantité de tyrosine libérée pendant la digestion.

Les résultats obtenus sont concrétisés par les histogrammes des figures 2 et 3 . Le; lactobacilles mésophiles, homo ou hétéro-fermentaires, ont une activité protéolytique limitée ; ils libèrent entre 0 et $12 \mu \mathrm{g}$ de tyrosine par ml d'extrait enzymatique et par heure de 


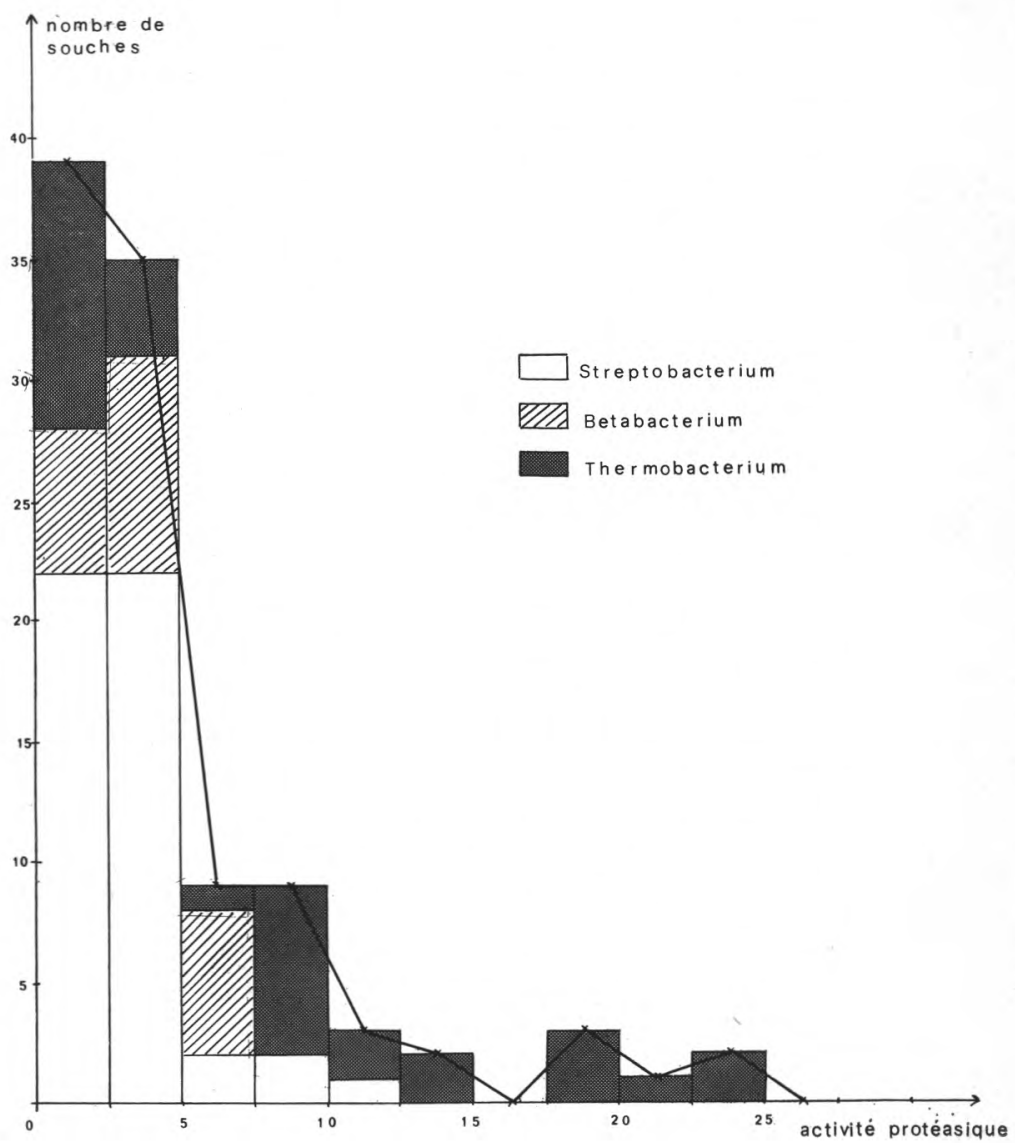

fig. 2

Distribution des souches de lactobacilles en fonction de leur activité protéolytique liée au cytoplasme

- En abscisses : les classes d'activité protéolytique ; l'activité est exprimée en $\mu \mathrm{g}$ de tyrosine libérée par $\mathrm{ml}$ de préparation enzymatique $\mathrm{E}_{2}$ et par heure.

- En ordonnées : le nombre de souches appartenant aux différentes classes; dans chaque classe, les parties diversement hachurées ou claires représentent le nombre de souches appartenant aux groupes Strepto-, Beta- et Thermobacterium. 

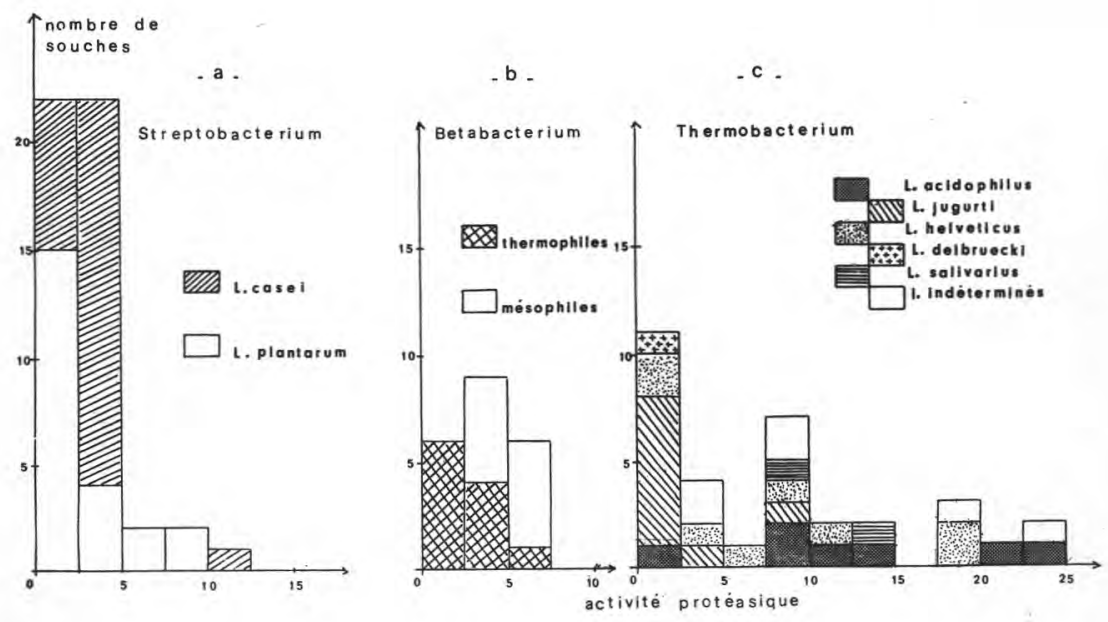

fig. 3

Répartition de l'activité protéolytique cytoplasmique chez différentes espèces de lactobacilles

- En abscisses : les classes d'activité protéolytique ; celle-ci est exprimée en $\mu \mathrm{g}$ de tyrosine libérée par $\mathrm{ml}$ de préparation enzymatique $\mathrm{E}_{2}$ et par heure.

- En ordonnées : le nombre de souches appartenant aux différentes classes ; dans chaque classe, les parties claires ou diversement hachurées représentent le nombre de souches de chaque espèce.

digestion, et ce résultat extrême n'est le fait que d'une seule souche de $L$. casei. L'espèce $L$. plantarum se caractérise notamment par son très faible pouvoir protéasique, certaines souches en étant d'ailleurs totalement dépourvues ; 60 p. 100 des souches appartiennent à la classe d'activité inférieure à 2,5 $\mu \mathrm{g}$ de tyrosine libérée.

L'examen des différents histogrammes met en évidence l'activité protéolytique plus marquée des lactobacilles du groupe Thermobacterium, à l'exception de l'espèce L. jugurti : 60 p. 100 des lactobacilles thermophiles libérant une quantité de tyrosine inférieure à $2,5 \mu \mathrm{g}$ appartiennent à cette espèce ; une souche, sur les neuf étudiées, est cependant douée d'un pouvoir protéasique appréciable.

Parmi les bactéries hétéro-fermentaires, l'espèce $L$. fermenti est très faiblement protéolytique. Ces lactobacilles ont une activité pratiquement identique à celle des Streptobacterium, notamment L. casei. 
TABLEAU 4. - Activité protéolytique endocellulaire des lactobacilles thermophiles

(activités exprimées en $\mu \mathrm{g}$ de tyrosine libérée par ml d'extrait et par heure)

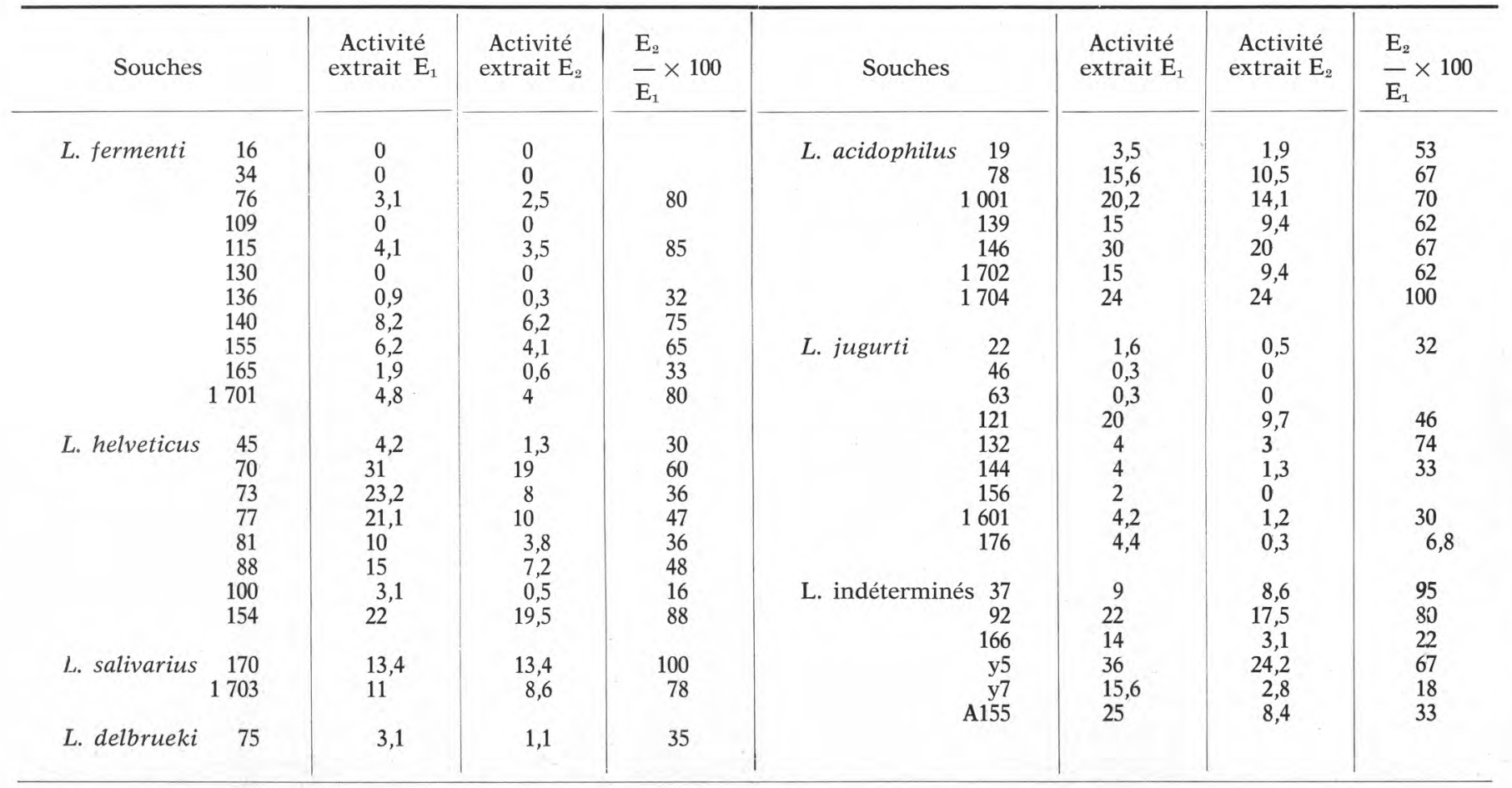

$E_{1}=$ Enzymes endocellulaires totales (cytoplasmiques + de surface).

$\mathrm{E}_{2}=$ Enzymes endocellulaires cytoplasmiques. 
3.2.3. Activité endocellulaire totale : Elle a été déterminée sur 44 lactobacilles thermophiles, homo ou hétéro-fermentaires, par la mesure de la quantité de tyrosine libérée.

L'extrait $\mathrm{E}_{1}$ contient les débris de la paroi cellulaire. Son activité sera donc la somme des activités des enzymes endocellulaires vraies ou cytoplasmiques et des enzymes liées à la paroi ou enzymes de surface (Pollock, 1962). Pour une même souche, la comparaison entre les quantités de tyrosine libérée par les extraits enzymatiques $\mathrm{E}_{1}$ et $\mathrm{E}_{2}$ permet donc d'apprécier l'importance de l'activité enzymatique liée à la paroi.

Les résultats obtenus pour cette activité intracellulaire totale (tab. 4) montrent que, dans les conditions de broyage choisies, une partie de l'activité protéolytique est due aux enzymes liées aux parois cellulaires. Le pourcentage de l'activité enzymatique cytoplasmique par rapport à l'activité endocellulaire totale varie considérablement suivant les souches : ainsi 16 p. 100 seulement de l'activité totale est due à ces enzymes dans le cas de L. helveticus souche 100 , et ce pourcentage atteint $88 \mathrm{p} .100$ pour L. helveticus souche 154,100 p. 100 pour L. acidophilus souche $1704 \ldots$

Cette particularité n'est pas un caractère lié à l'espèce. Au sein d'une même espèce, nos résultats montrent qu'il existe une très grande variation dans l'activité relative des enzymes de surface.

3.2.4. Nature de l'activité protéolytique : Les essais préliminaires ont montré que les produits libérés sont solubles dans l'acide trichloracétique à 2 et à $12 \mathrm{p} .100$. Ceci permet de penser que les enzymes protéolytiques des lactobacilles libèrent des composés azotés de faible poids moléculaire. Cette hypothèse a été vérifiée par le dosage des groupements $-\mathrm{NH}_{2}$ libres dans le filtrat.

Dans cette expérience, 33 lactobacilles thermophiles ont été étudiés. Sur le même filtrat trichloracétique à 2 p. 100 , les produits de dégradation ont été estimés selon les deux méthodes : méthode de Anson et colorimétrie avec le réactif à la ninhydrine. leu

Il est alors possible d'établir pour chaque souche, les rapports $\frac{1}{\text { tyr }}$ pour les extraits enzymatiques $E_{1}$ et $E_{2}$; la valeur de ce rapport concrétise le mode d'action des enzymes protéolytiques, une valeur élevée étant l'indice d'une activité de type exopeptidasique.

En effet la méthode de Anson ne fait qu'apprécier le nombre de molécules de caséine dégradée, le poids moléculaire des produits libérés n'intervenant pas ; en revanche, la méthode à la ninhydrine permet d'estimer le nombre de liaisons peptidiques rompues et, pour un même nombre de molécules de caséine dégradée, les résultats seront d'autant plus élevés que les produits libérés seront de poids moléculaire plus faible. 
TABLEAU 5

Caractères des enzymes protéolytiques endocellulaires de 33 lactobacilles thermophiles

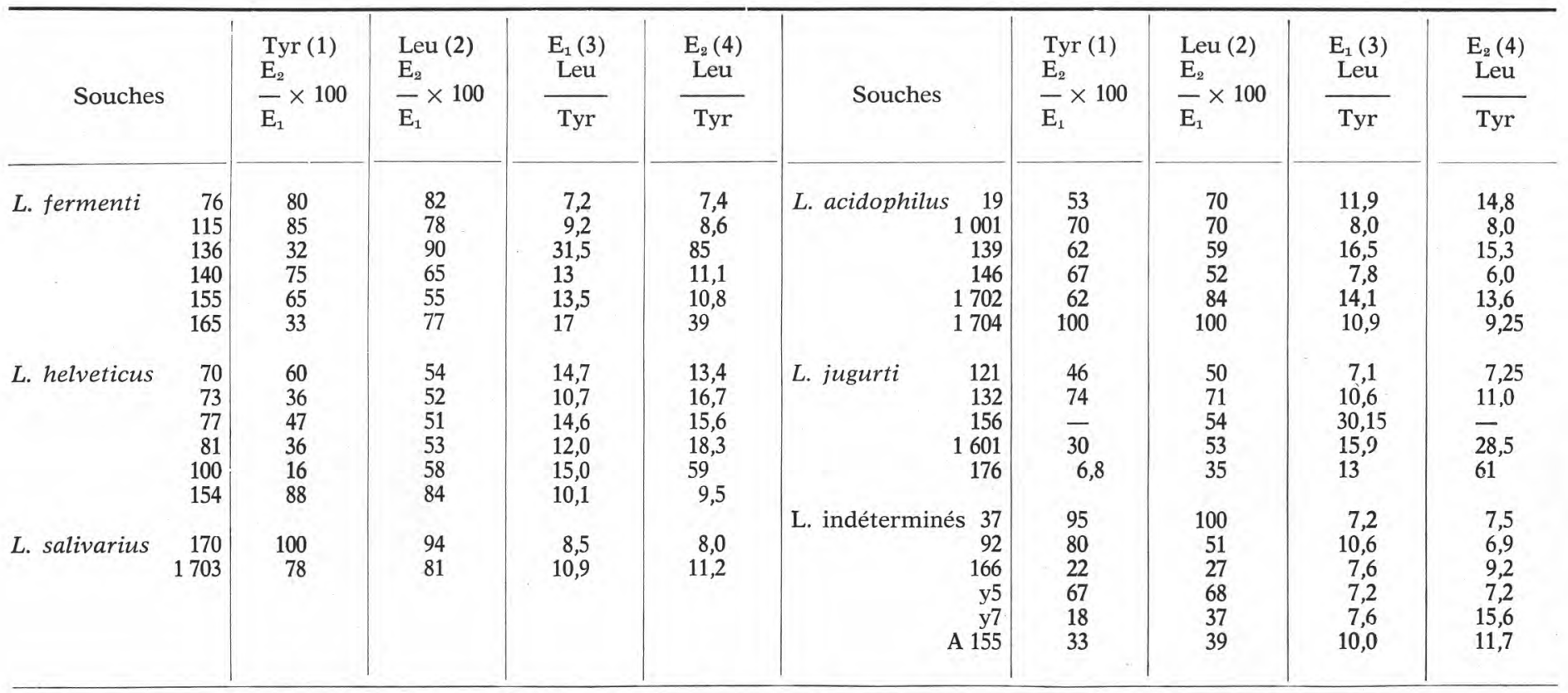

(1) Pourcentage de l'activité cytoplasmique par rapport à l'activité totale (activités exprimées en tyrosine).

(2) Pourcentage de l'activité cytoplasmique par rapport à l'activité totale (activités exprimées en leucine).

(3) Rapport $\frac{\text { Leu }}{\text { Tyr }}$ de l'extrait endocellulaire total.

(4) Rapport $\frac{\text { Leu }}{\text { Tyr }}$ de l'extrait endocellulaire cytoplasmique. 
Les résultats obtenus figurent dans le tableau 5. L'examen des valeurs trouvées chez les lactobacilles $\left(7,1<\frac{\text { leu }}{\text { tyr }} \mathrm{E}_{1}<31,5\right)$ et leur comparaison avec celles trouvées chez d'autres micro-organismes doués d'une activité de type endopeptidasique, montrent que les lactobacilles libèrent préférentiellement de petits peptides ou des acides aminés. Ainsi, dans des conditions de digestion et de dosage identiques, le rapport $\frac{\text { leu }}{\text { tyr }}$ de la protéase majeure de Penicillium caseicolum est seulement de 0,6.

La population étudiée semble très hétérogène et cette hétérogénéité se manifeste par une variation de 1 à 4 dans la valeur des rapports $\frac{\text { leu }}{\text { tyr }}$ pour l'extrait $E_{1}$; les souches présentent donc une aptitude différente à la libération préférentielle d'acides aminés et cette constatation est encore plus évidente à l'examen des résultats obtenus avec $E_{2}$ : les valeurs extrêmes sont en effet 6,0 pour $L$. acidophilus souche 146 , et 85 pour $L$. fermenti souche 136 , soit une variation de 1 à 12 suivant les souches.

Il semble donc que les extraits enzymatiques $\mathrm{E}_{1}$ et $\mathrm{E}_{2}$ puissent avoir un mode d'action différent, l'extrait endocellulaire cytoplasmique étant doué d'un pouvoir exopeptidasique beaucoup plus marqué. C'est le cas notamment chez L. helveticus souche 100, L. jugurti souche 176 et L. fermenti souches 136 et 165 .

Remarquons cependant que de telles différences ne sont trouvées que chez un nombre limité de souches, les rapports $\frac{\text { leu }}{\text { tyr }}$ pour $\mathrm{E}_{1}$ et pour $\mathrm{E}_{2}$ étant dans la majorité des cas très voisins.

\section{4) Discussion}

Deux remarques préliminaires s'imposent qui limiteront les conclusions que l'on peut tirer des résultats obtenus :

- Les conditions expérimentales choisies pour la production d'enzymes et pour la détermination de l'activité ne sont probablement pas les conditions les plus favorables pour apprécier l'aptitude à la protéolyse de toutes les souches étudiées. Le protocole a été adopté dans le but de faire une étude comparative du pouvoir protéolytique d'une population de lactobacilles et non pas de déterminer l'aptitude réelle optimale de chaque souche ; il devait donc être identique pour tous les micro-organismes et répondre aux impératifs de l'étude : mettre en évidence des activités susceptibles d'être très dispersées. Sa mise au point a été réalisée à partir d'essais préliminaires dont les résultats sont en accord avec ceux de diffé- 
rents chercheurs ayant travaillé sur ces mêmes bactéries (Baribo et Foster, 1952 ; Ducastelle et Lenoir, 1969).

- L'étude a porté sur un échantillonnage complexe puisque la population se compose de 10 espèces et de quelques lactobacilles non identifiés. Le nombre restreint de souches et la diversité des espèces ne permettent donc pas de conduire une étude statistique mettant en évidence l'homogénéité ou l'hétérogénéité de la population pour le caractère considéré. Ce travail permet seulement de porter une appréciation sur les capacités respectives des souches, éventuellement des espèces, à dégrader les protéines, notamment la caséine.

La courbe de répartition des individus en fonction de leur activité protéolytique cytoplasmique ( $E_{2}$ ) (fig. 2) montre que 75 p. 100 des bactéries étudiées ont une activité inférieure à l'activité moyenne de la population ( $\bar{x}=6,5 \mu \mathrm{g}$ de tyrosine par ml d'extrait et par heure de digestion). Les espèces dominantes dans ces classes d'activité restreinte sont : $L$. casei, $L$. plantarum, $L$. fermenti et $L$. jugurti. Parmi les micro-organismes doués d'une aptitude à la protéolyse supérieure à la moyenne, 68 p. 100 des individus appartiennent au groupe Thermobacterium. Toutefois, au sein d'une même espèce, les souches possèdent des activités différentes et l'aptitude à la protéolyse ne semble pas être un caractère lié à l'espèce.

Dans les conditions de broyage utilisées, une partie plus ou moins importante de l'activité semble due à des enzymes liées à la paroi cellulaire et l'importance de cette activité varie selon les souches. Dans la majorité des cas, ces enzymes semblent posséder le même caractère d'activité que les enzymes cytoplasmiques, les leu

rapports - pour $E_{1}$ et $E_{2}$ étant identiques (tab. 5). Toutefois avec six souches, (L. helveticus 100, L. jugurti 1601 et 176, L. fermenti 136 et 165 et la suuche non identifiée $y_{7}$ ), les valeurs des rapports leu

tyr

cytoplasmique possède une activité exopeptidasique nettement plus élevée que celle qui peut être attribuée au système enzymatique lié à la paroi. Chez certaines souches, il semble donc y avoir existence de deux systèmes protéolytiques distincts, localisés dans des organites cellulaires différents et caractérisés par une aptitude plus ou moins marquée à la libération d'acides aminés.

Cependant ce caractère d'hétérogénéité au niveau du type d'activité des systèmes enzymatiques ne peut être mis en évidence que si l'activité des enzymes cytoplasmiques est faible par rapport à l'activité endocellulaire totale. L'activité des enzymes de surface est en effet évaluée par différence entre l'activité globale et l'activité cytoplasmique et, si cette dernière est majeure, elle peut masquer le 
type d'activité des enzymes de surface. Les six souches citées ci-dessus répondent à cette exigence.

Une telle caractéristique se retrouve également chez deux souches non identifiées 166 et A 155 , mais les valeurs $\frac{\text { leu }}{\text { tyr }}$ pour $E_{1}$ et $\mathrm{E}_{2}$ sont alors pratiquement identiques ; ces souches synthétisent probablement deux systèmes protéolytiques distincts mais ayant un mode d'action semblable.

Enfin, dans la majorité des cas, l'activité protéasique cytoplasmique représente 50 à 100 p. 100 de l'activité endocellulaire totale. Le mode d'action des deux systèmes enzymatiques (s'ils existent) ne peut alors être comparé par cette méthode. L'étude d'un extrait enzymatique constitué par les enzymes de surface elles-mêmes sera nécessaire pour connaître leur type d'activité.

Il apparaît finalement que les lactobacilles constituent une population très hétérogène pour le caractère " aptitude à la protéolyse ". Cette hétérogénéité se manifeste non seulement entre les espèces, mais également entre les souches appartenant à la même espèce. Elle porte à la fois sur l'intensité de la production enzymatique, sur la localisation des systèmes enzymatiques au sein de la cellule et sur le caractère d'activité des enzymes dont la nature exopeptidasique est plus ou moins accentuée.

Toutefois les lactobacilles thermophiles sont doués d'une activité protéasique plus élevée que celle des autres espèces. Ces microorganismes, qui constituent la flore dominante des levains des fromages à pâte cuite mais qui disparaissent au cours de la maturation, sont donc susceptibles de libérer dans la pâte leurs enzymes intracellulaires et de participer ainsi à la dégradation de la caséine et à la libération de produits susceptibles de constituer les éléments de l'arôme et de la saveur. Il est vraisemblable que c'est à leur action que le Gruyère de Comté doit sa richesse caractéristique en oligopeptides et en acides aminés (Lenoir, 1963 ; Do Ngoc, Lenoir, Choisy, 1971). L'activité exopeptidasique des lactobacilles expliquerait également le rôle stimulant de ces micro-organismes sur Streptococcus thermophilus (Accolas, Veaux, Auclair, 1971).

\section{Résumé et conclusions}

Dans les fromages à maturation lente, les lactobacilles qui représentent à certaines périodes la microflore dominante, sont susceptibles de jouer un rôle dans la dégradation de la caséine et de participer ainsi à la formation de la saveur et de l'arôme du fromage. Or l'aptitude réelle de ces micro-organismes à la production d'enzymes protéolytiques est encore mal connue, peu d'études systématiques portant sur un grand nombre de souches ayant été réalisées.

La mise en place d'une collection de lactobacilles appartenant à 10 espèces différentes, isolés de lactosérums ou de fromages dans 
lesquels ces bactéries représentent la population principale, et la détermination de l'activité protéolytique endocellulaire de 103 souches ont permis de caractériser ces micro-organismes suivant leur aptitude à la protéolyse.

Dans les conditions de production et de digestion choisies, la population est très hétérogène et cette hétérogénéité porte à la fois sur l'activité protéolytique, sur la localisation des enzymes à l'intérieur de la cellule et sur la nature des composés libérés. L'aptitude à la protéolyse ne semble pas être une caractéristique d'espèces mais de souches. Toutefois les souches les plus actives appartiennent au groupe Thermobacterium (Orla-Jensen). Les enzymes synthétisées ont une activité de type exopeptidasique et libèrent préférentiellement des composés de faible poids moléculaire. De nombreuses souches possèdent une activité protéasique due en partie à la présence d'enzymes de surface.

Dans certaines fabrications fromagères, les enzymes libérées par la lyse des lactobacilles pourraient intervenir activement dans le processus de dégradation de la caséine par libération d'acides aminés et d'oligopeptides.

\section{S u m m a ry}

In slowly ripening cheeses, lactobacilli which represent at some periods an important fraction of bacterial flora are susceptible to take a part in the breakdown of casein and to participate so to the formation of the taste and the aroma.

A collection of strains of lactobacilli from 10 various species isolated from lactoserums or cheeses in which those microorganisms are the dominant bacterial population has been constituted. The determination of endocellular proteolytic activity of 103 strains has permitted to class those microorganisms according to proteasic ability.

The population is very incongruous. The heterogeneity is due to :

- the importance of proteolytic activity, which varies between 0 and $36 \mu \mathrm{g}$ of free tyrosin per $\mathrm{ml}$ of extract and per hour of digestion, enzyms,

- the relative activity of cytoplasmic enzyms and surface-bound

- the composition of constituents that were set free by enzymatic activity.

The proteolytic ability cannot probably be attributed to species but rather to strains. Nevertheless, the most active strains belong to Thermobacterium (Orla-Jensen). Enzyms possess an exopeptidasic activity. In a few cheese makings, cell- bound enzyms, liberated by the cellular lysis, are able to degrad casein and release oligo-peptides and amino-acids. 


\section{Références bibliographiques}

Accolas (J. P.), Veaux (M.), Auclair (J.) (1971). - Etude des interactions entre diverses bactéries lactiques thermophiles et mésophiles, en relation avec la fabrication des fromages à pâte cuite. Lait, 505-506, 249-272.

Amunstad (O.) (1950). - Une étude de l'activité protéolytique de la présure et de quelques bactéries avec une référence spéciale à l'affinage des fromages (en suédois). Medd. Mejeriförs, Malmö, 28, 170 p.

AnNibaldi (S.) (1969). - Ricerche elettroforetiche sull' attiva' caseinolitica di Lactobacillus jugerti. Scienza Tec. latt. casear, 20, 103-109.

ANSoN (M. L.) (1938). - The estimation of pepsin, papain and cathepsin with hemoglobulin. J. Gen. Physiol., 22, 79.

BARIBo (L. E.), Foster (E. M.) (1952). - The intracellular proteinases of certain organisms from cheese and their relationship to the proteinases in cheese. J. Dairy Sci, 35, 149-150.

BottazzI (V.) (1959). - Studio sulla microflora del formaggio Fontina. I. La microflora lattica durante il processo di maturazione. Latte, 33, 675-678.

Brandsaeter (E.), Nelson (F.E.) (1956). - Proteolysis by Lactobacillus casei I. Proteinase activity. J. Bact, 72, 68-72.

Carini (S.), Ottogalli (G.) (1967). - L'attivita caseinolitica del batteri lattici. Annali. Microbiol, 17, 85-92.

Christophersen (J.), Thiele (H.) (1952). - Influence of medium and temperature on the proteolytic activities of cheese bacteria. Kieler milchw. Forsch Ber., 4, 683-700.

DolezaleK (J.) (1966). - Proteolytic activity of Streptococcus lactis, Streptococcus cremoris, Streptococcus diacetylactis, Lactobacillus helveticus, Lactobacillus casei. C.R. XVII Congrès International de Laiterie, Munich, D, 523-526.

Do Ngoc (M.), Lenoir (J.), Choisy (C.) (1971). - Les acides aminés libres des fromages affinés de Camembert, Saint-Paulin et Gruyère de Comté. Rev. Lait. Fr., 288, 447-462.

Ducastelle (A.), Lenoir (J.) (1969). - Contribution à l'étude de la flore microbienne du fromage de type Saint-Paulin. Lait, 489-490, 615-636.

Evans (J. B.), Niven (C. F.) (1951). - Nutrition of the hetero fermentative lactobacilli that cause greening of cured meat products. J. Bact., 62, 599-603.

Feagan (J. T.), Dawson (D. J.). (1959). - Bacteriology of Cheddar cheese. Some observations on the microflora during maturation. Austr. J. Dairy Technol., 14, 59-66.

Folin (O.), Ciocalteu (V.) (1927). - On tyrosine and tryptophane determination in proteins. J. Biol. Chem., 73, 627.

JoHns (C. K.), Cole (S. E.) (1959), - Lactobacilli in Cheddar cheese. J. Dairy Res., 26, 157-161.

LENOIR (J.) (1963). - Note sur la composition en matières azotées des fromages affinés des types Camembert, Saint-Paulin et Gruyère de Comté. Ann. Technol. Agric., 12, 53-57.

Lusiani (G.), Bianchi-Salvadori (B.), Salvadori (P.) (1971). - Activité protéolytique de quelques micro-organismes responsables de la maturation des fromages persillés. Lait, 51 (507), 431-436.

MabittT (L. A.), ZielinsKa (M.) (1956). - The use of a selective medium for the enumeration of lactobacilli in Cheddar cheese. J. appl. Bact., 19, 95-101.

De Man (J. C.), Rogosa (M.), Sharpe (M. E.) (1960). - A medium for the cultivation of lactobacilli. J. appl. Bact., 23, 130-135. 
MatTson (S.) (1965). - The determination of the configuration of lactic acid in bacterial cultures. Milk and Dairy Research, Report $\mathrm{n}^{\circ} 71$.

MilleR (I.), KANDLER (O.) (1967). - Eiweissabbau und anreicherung freier aminosäuren durch milchsäurebakterien in milk. I. Die veränderung der stickstofffraktionnen. Milchwissenschaft, 22, 150-159.

Moore (S.), STEIN (W. H.) (1948). - Photometric ninhydrine methode for use in the chromatography of amino-acids. J. Biol. Chem., 176, 367-388.

NAYLoR (J.), Sharpe (M. E.) (1958). - Lactobacilli in Cheddar cheese. I. The use of selective media for isolation and of serological typing for identification. J. Dairy Res., 25 (1), 92-103.

OhmiYa (K.), Sato (Y.) (1968). - Studies on the proteolytic action of dairy lactic acid bacteria. 4. Changes of the casein treated with Lactobacillus bulgaricus, Lactobacillus helveticus, or Streptococcus lactis. Agr. biol. Chem., 32, 291-296.

OhmiYa (K.), Sato (Y.) (1969), - Studies on the proteolytic action of dairy lactic acid bacteria. 7. Action of intracellular protease of Lactobacillus bulgaricus, Lactobacillus helveticus or Streptococcus lactis on casein. Agr. biol. Chem., $33,669-675$.

ORLA-JENSEN (1919). - The lactic acid bacteria. Andr. Fred. Host and Son, Copenhague.

Perry (K. D.), Sharpe (M. E.) (1960). - Lactobacilli in raw milk and in Cheddar cheese. J. Dairy Res., 27, 267-275.

Pollock (M. R.) (1962). - Dans Gunsalius et Stanier, The bacteria, vol. 4, 121178. Academic Press, New-York.

RAPP (M.) (1969). - Uber das eiweissabbauvermögen von milchsäurebakterien. Milchwissenschaft, 24, 208-211.

REUTER (G.) (1970). - Laktobazillen und erg verwandte mikroorganismen in fleisch und fleischwaren. Die Fleischwirtschaft (7), 951-962.

Rogosa (M.), SHARPE (M. E.) (1959). - An approach to the classification of the lactobacilli. J. appli. Bact., 22, 329.

SAHLI (K. W.), LehmanN (W.) (1959). - Etude bactériologique et chimique sur la maturation des fromages d'Emmental de haute qualité. Schweiz. Milchztg, 85 (10), 505-512.

Sato (Y), NaKashima (I.) (1965). - Etude de l'action protéolytique des bactéries lactiques. I. Préparation par ultra-sons des protéases intracellulaires et leur action sur la caséine. Agric. biol. Chem., 29, A 29.

Sharpe (M. E.) (1962). - Taxonomy of the lactobacilli. Dairy Sci. Abstr., 24, 109-118.

Steinsholt (K.), Calbert (H. E.) (1960). - A rapid colorimetric method for the determination of lactic acid in milk and milk products. Milchwissenschaft, 15, 7-11.

SyrJANEN (H.) (1965). - Lactobacilles dans le fromage d'Edam. Karjantuote, $48,487-489$.

Technicon (1968). - Monograph 3. 6th Colloquium in amino acid analysis, 162-163.

Tofte JESPERSEN (N. J.). (1966). - Proteolytic activity of some lactic acid bacteria. C.R. XVII Congrès International de Laiterie. Munich, D, 465-470. 